\title{
Mielolipoma extradrenal de localización presacra. A propósito de un caso
}

\author{
F. J. Torres Gómez, F. J. Torres Olivera, A. García
}

\section{Resumen}

Los mielolipomas son tumores benignos constituidos por elementos hematopoyéticos en diferentes grados madurativos y sin alteraciones histológicas combinados en diferentes proporciones con tejido adiposo maduro. Son tumores generalmente asintomáticos si bien en la mitad de los casos se asocian a síntomas, consistentes en dolor abdominal, hematuria, masa palpable e hipertensión. Los mielolipomas presentan la mayoría de las veces una localización adrenal siendo la localización presacra poco frecuente. El diagnóstico diferencial hay que establecerlo clínicamente con otros tumores benignos y malignos de localización retroperitoneal e histológicamente con la hematopoyesis extramedular.

Presentamos el caso de un paciente de 54 años con un mielolipoma asintomático de localización presacra.

\section{Palabras clave:}

Mielolipoma. Localización presacra. Extradrenal.

Oncología, 2005; 28 (6):304-307 


\section{Summary}

Myelolipomas are benign tumor composed of mature and immature hematopoietic elements without histologic alterations admixed with mature adipose tissue in different proportions. Symptoms are only present in one half of the patients consisting in abdominal pain, hematuria, palpable mass and hypertensión. Myelolipomas occur most often in the adrenal glands. Presacral extraadrenal location is rare. The differential diagnosis is prompted clinically with other retroperitoneal benign and malignant tumors and histologically with extramedullary hematopoiesis.

We present the case of a 54 year old patient with an asymptomatic myelolipoma of presacral location.

Key words: Myelolipoma. Presacral location. Extraadrenal

\section{Introducción}

Los mielolipomas son tumores benignos compuestos por elementos hematopoyéticos en diferentes estadios madurativos y sin alteraciones histológicas en combinación con tejido adiposo. Aunque algunos tumores muestran predominio del componente hematopoyético, en la mayoría de los casos es el componente adiposo el que mayor proporción representa. Las glándulas suprarrenales son con mucho la localización más común, registrándose cifras de hasta $0,4 \%$ de autopsias. En algunos casos estas son afectadas bilateralmente. Por el contrario, no son muchos los casos de mielolipomas descritos en localización extraadrenal ${ }^{4,11-13}$; de ellos, aproximadamente la mitad presenta una ubicación presacra. Le siguen en frecuencia el retroperitoneo y la pelvis, aunque también han sido descritas localizaciones tan diversas como mediastino ${ }^{4}$, pulmón ${ }^{1,3}$, estómago, hígado, meninges y riñón ${ }^{10}$. Presentamos el caso de un mielolipoma de localización presacra sin cuadro hematológico acompañante.

\section{Descripción del caso}

Paciente de 54 años que en el transcurso de una exploración ginecológica rutinaria se descubre por ecografía una tumoración de $6 \mathrm{~cm}$ de diámetro máximo sonodensa, homogénea y mal definida en sus contornos posteriores ya que no existe interfase con el tejido graso circundante, que presenta una locali- zación presacra, detrás de la imagen del recto-sigma. Dicha masa fue confirmada por TAC en la que se apreció una lesión redondeada, bien delimitada, de unos $6 \mathrm{~cm}$ de diámetro, que presentaba coeficientes de absorción predominantemente grasos con discreta heterogeneidad, todo ello compatible con posible tumor dermoide presacro. Dicha paciente presentaba unas cifras de hematocrito de $38 \%$ sin alteraciones hematológicas acompañantes. No se apreciaron visceromegalias.

Macroscópicamente se trataba de una tumoración irregular bien delimitada de $7 \times 5 \times 3 \mathrm{~cm}$ que parecía estar constituida fundamentalmente por tejido adiposo, observándose un área central hemorrágica de $2 \mathrm{~cm}$, y en la periferia, una serie de áreas blanquecinas de aspecto fibroso así como otras edematosas (Fig. 1).

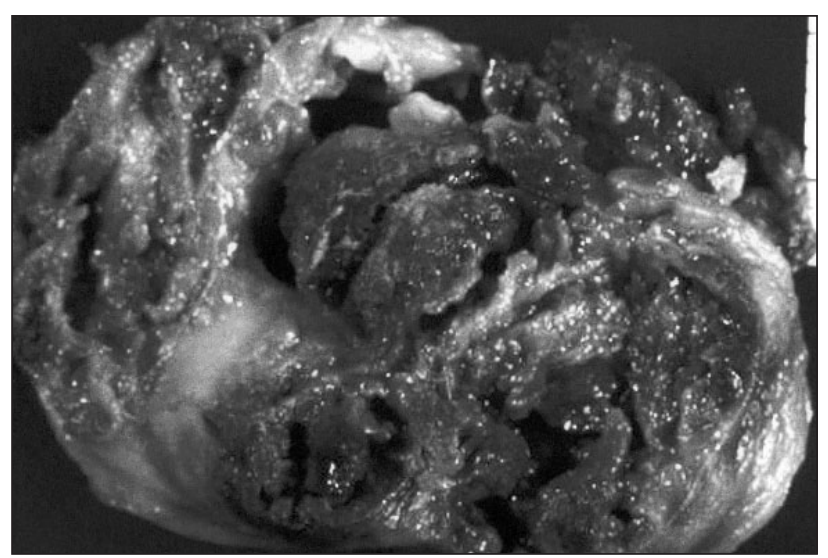

Figura 1. Mielolipoma extraadrenal. Aspecto macroscópico. 


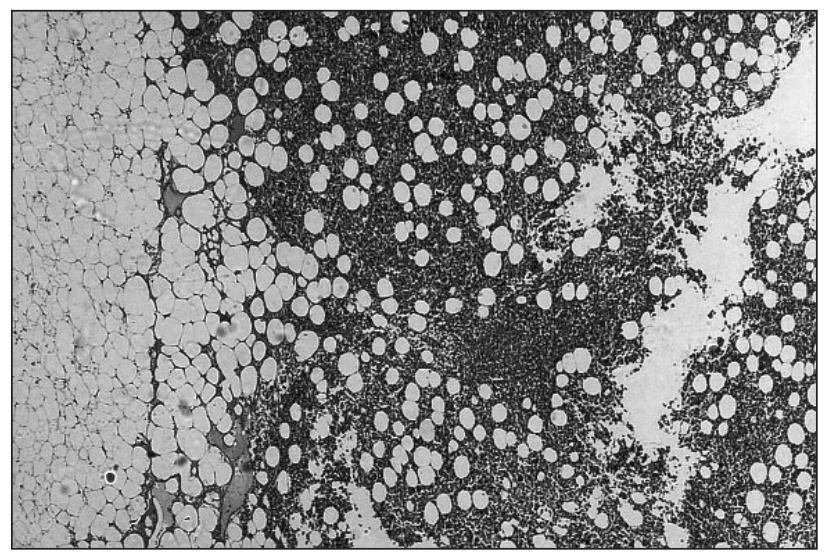

Figura 2. Histología. Combinación de tejido adiposo con elementos hematopoyéticos. HE.5x

Histológicamente la neoplasia se encontraba relativamente bien circunscrita y estaba constituida por tejido adiposo maduro, tejido conectivo laxo y celularidad hematopoyética mixta (Fig. 2). Este último componente predominaba en las áreas centrales del tumor y se hallaba representado por las tres series hematopoyéticas (megacariocítica, serie roja y serie blanca) (Fig. 3), con diferentes proporciones según la zona estudiada y con un índice eritromieloide que oscilaba entre $1: 1$ y $2: 1$. Los megacariocíticos se observaban con facilidad y aunque se encontraron ocasionales formas inmaduras de los mismos, no presentaban rasgos displásicos. El estroma de las áreas hematopoyéticas era casi exclusivamente de tipo adiposo. En la zona más periférica del tumor predominaba el tejido adiposo maduro con focos de fibrosis y abundantes hemosideri-

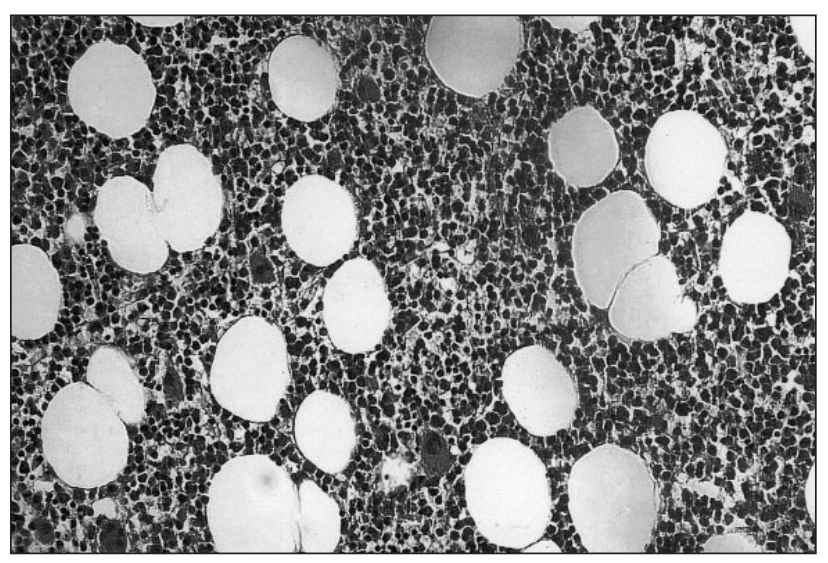

Figura 3. Histología. Vacuolas adiposas y elementos de las tres series hematopoyéticas. HE.40x. nófagos. No se observaron lipoblastos ni células mesenquimales bizarras y existía cierto componente inflamatorio de predominio linfocitario, ocasionalmente dispuesto en agregados de distribución aleatoria tanto en las zonas hematopoyéticas como en las adiposas.

\section{Discusión}

Existen cuatro patrones clinicopatológicos en los cuales pueden ser encuadrados los mielolipomas ${ }^{5}$ : localización adrenal aislada, localización adrenal con hemorragia, localización adrenal con asociación a patología adrenal y localización extraadrenal, constituyendo esta última, como en nuestro caso, una situación relativamente poco frecuente.

Se trata de tumores por lo general asintomáticos que se suelen diagnosticar en el transcurso de estudios de imagen dirigidos a otras etiologías siendo difíciles de identificar en la radiografía plana ${ }^{2}$ a menos que tengan gran tamaño o estén constituidos principalmente por tejido adiposo. En cambio la ecografía muestra heterogeneidad debido a su arquitectura no uniforme constituyendo un método diagnóstico eficaz para su identificación. La TAC y la RMN constituyen asimismo pruebas eficaces para el estudio de dichas lesiones.

Existen en la literatura referencias a la utilización de la PAAF como método diagnóstico de primera linea para la catalogación del mielolipoma ${ }^{6,8}$ (se recomienda la tinción de Romanowsky para poner de manifiesto con mayor claridad los elementos hematopoyéticos) si bien habrá que recurrir a la cirugía en casos dudosos o en aquellos que se acompañan de sintomatología. En estos casos es de gran ayuda el citodiagnóstico intraoperatorio mediante improntas o raspado de la pieza quirúrgica, influyendo en la conducta a seguir en quirófano ${ }^{9}$.

El diagnóstico diferencial de los mielolipomas debe realizarse principalmente con los tumores hematopoyéticos extramedulares ${ }^{13}$ los cuales se suelen asociar a anemia severa y cuadros mieloproliferativos así como a hepato y esplenomegalia y suele tratarse de focos múltiples, de localización predominantemente mediastínica mientras que los mielolipomas suelen tener una localización única, están bien delimitados y su localización es predominantamente abdominal. Además, ambas entidades presen- 
tan características histológicas que ayudan a su distinción tales como el predominio de elementos hematopoyéticos, la hiperplasia eritroide y la falta de agregados linfoides, presentes en los tumores hematopoyéticos extramedulares y ausentes en los mielolipomas.

\section{Bibliografía}

1. Franiel T, Fleischer B, Raab BW, Fuzesi L. Bilateral thoracic extraadrenal myelolipoma. Eur J Cardiothorac Surg 2004 Dec; 26(6):1220-2.

2. Bilateral extraadrenal perirenal myelolipomas: an imaging challenge. AJR Am J Roentgenol.2004 Sep;183(3):833-6.

3. Spanta R, Saleh HA, Khatib G. Fine needle aspiration diagnosis of extraadrenal myelolipoma presenting as a pleural mass. A case report. Acta Cytol 1999 Mar-Apr;43(2):2958.

4. Minamiya Y, Abo S, Kitamura M, Izumi K. Mediastinal extraadrenal mielolipoma: report of a case. Surg Today 1997; 27(10):971-2.

5. Rao P, Kenney PJ, Wagner BJ. Imaging and pathologic features of myelolipoma. Radiographics 1997 NovDec;17(6):1373-85.

6. Bryan JA, Sykes CH, Garvin DF. Fine needle aspiration diagnosis of a mesenteric myelolipoma: a case report. Acta Cytol 1996 May-Jun;40(3):592-4.

7. Hunter SB, Schemankewitz EH, Patterson C, Yarma VA. Extraadrenal myelolipoma. A report of two cases. Am J Clin Pathol 1992 Mar;97(3):402-4.

8. Evans GW, Olinde HD, Kozdereli E. Extraadrenal myelolipoma. A lesion that can be diagnosed by fine needle aspiration biopsy. Acta Cytol 1990 Jul-Aug;34(4):536-8.

9. Extraadrenal myelolipoma. Intraoperative cytodiagnosis on touch preparations. Acta Cytol 1990 Jan-Feb:34(1):89-91.

10. Mitual B. Amin, MD; Satish K. Tickoo, MD; Daniel Schultz, MD; Mahul B. Amin, MD. Myelolipoma of the Renal Sinus. Arch Pathol Lab Med 1999; 123:631-634.

11. Grignon DJ, Shkrum MJ, Smout MS. Extraadrenal Myelolipoma. Arch Pathol Lab Med 1989; 113: 52-54

12. Jagadha V, Ramaswamy G. Extraadrenal Myelolipoma. NYS J Med 1985; 60

13. Chen KT, Felix EL, Flam MS. Extraadrenal Myelolipoma. Am J Clin Pathol 1982 Sep; 78(3):386-9 